\title{
Adolescentes chilenos: compra, valores materiales y satisfacción con la vida ${ }^{1}$
}

\section{Chilean Teenagers: Consumption, Material Values and Life Satisfaction}

\author{
Luis Mario Castellanos Alvarenga (*) luismario happy@hotmail.com \\ Marianela Denegri Coria (*) marianela.denegri@ufrontera.cl \\ Pamela Alejandra Salazar Valenzuela (*)pamela.salazar.v@ufrontera.cl \\ (*) Universidad de La Frontera \\ (Recibido: 5 de septiembre de 2017; Aceptado para su publicación: 15 de marzo de 2018)
}

Cómo citar: Castellanos, L. M., Denegri, M. y Salazar, P. A. (2020). Adolescentes chilenos: compra, valores materiales y satisfacción con la vida. Revista Electrónica de Investigación Educativa, 22, e04, 1-11. https://doi.org/10.24320/redie.2020.22.e04.2155

\section{Resumen}

La globalización y el modelo económico neoliberal en Chile sitúa al consumo como articulador del proceso de construcción de identidad individual y colectiva, dando relevancia al materialismo y a la impulsividad en la compra, impactando especialmente en las generaciones que han crecido dentro del modelo. El presente estudio examina la relación entre estilos de compra, valores materiales y satisfacción con la vida en adolescentes de educación Secundaria de distintos niveles socioeconómicos del sur de Chile mediante un muestreo bietápico (423 estudiantes de primero y segundo año). Los resultados indican una relación directa y positiva entre materialismo, compra impulsiva y compra compulsiva; una relación inversa y baja entre satisfacción con la vida y compra compulsiva y valores materiales; una relación lineal entre compra impulsiva y nivel socioeconómico, y no se encontró relación entre nivel socioeconómico y satisfacción con la vida y materialismo.

Palabras clave: Estilos de compra, materialismo, satisfacción vital, nivel socioeconómico, adolescencia.

\section{Abstract}

Globalization and the neoliberal economic model in Chile have made consumption a cornerstone of individual and collective identity construction processes, attaching importance to materialism and impulse buying, which has particularly affected generations that have grown up inside this model. This study explores the relationship between styles of consumption, material values, and life satisfaction in high school students from different socioeconomic levels in southern Chile through two-stage sampling (423 first and second-year students). The findings show a direct, positive relationship between materialism, impulse buying and compulsive buying; a low inverse relationship between life satisfaction and compulsive buying and material values; and a linear relationship between impulse buying and

\footnotetext{
${ }^{1}$ Este estudio forma parte del proyecto FONDECYT 1150665 "Alfabetización financiera y económica, actitudes hacia el dinero, patrones de consumo y satisfacción vital en estudiantes chilenos. Hacia un modelo integrativo".
} 
socioeconomic level. No relationship was found between socioeconomic level and life satisfaction and materialism.

Keywords: Purchase styles, life satisfaction, material values, socioeconomic level, adolescence.

\section{Introducción}

En las últimas décadas la sociedad chilena ha experimentado importantes cambios productivos, tecnológicos, institucionales y sociales, dando como resultado un contexto sociopolítico y económicocultural cambiante y complejo (Gempp et al., 2007). Estos cambios impactaron en la dinámica de las relaciones sociales (Denegri y Martínez, 2004), transformando a una sociedad austera y tradicional en una sociedad de fuerte consumo y orientada al éxito individual, lo que impacta a todos los segmentos de la sociedad y especialmente a niños y adolescentes (Denegri, 2010).

En la adolescencia, la construcción del "yo" individual y grupal aparece profundamente ligado a las prácticas de consumo y a lo que éste representa en términos de prestigio, poder y acceso a mundos reales o soñados, homogeneizando las aspiraciones entre las distintas clases sociales (Denegri, 2010; Orcasita y Uribe, 2010). Al mismo tiempo, en esta etapa se consolidan las actitudes hacia el consumo y la compra y el nivel de materialismo, lo cual podría tener influencia en la forma en que los jóvenes evalúan su vida y la satisfacción con ésta (Shim et al., 2011).

En relación con los estilos de compra, algunos estudios señalan que estos pueden clasificarse en compra planificada, impulsiva y compulsiva. La compra planificada o compra cognitiva es aquella con alta activación cognitiva y baja activación emocional, ello la orienta a la búsqueda y contraste de información antes de tomar la decisión (Castellanos et al., 2016; Jurado et al., 2011; Luna y Quintanilla, 2000; Luna et al., 2004). El desarrollo estable de este estilo de compra genera resultados financieros positivos y permite disminuir la compra impulsiva y compulsiva (Denegri, 2010).

Por su parte, la compra impulsiva implica alta activación emocional, lo que se expresa en una tendencia a comprar de forma espontánea, irreflexiva e inmediata (Jurado et al., 2011; Lai, 2010; Rook y Fisher, 1995) siendo un predictor de la compra compulsiva (Vogt et al., 2015). Esta última es considerada patológica y se caracteriza por un impulso irresistible de comprar con alta presencia de ansiedad y pérdida de control sobre el comportamiento a pesar de las consecuencias negativas en la vida personal, social o laboral y en su equilibrio financiero (Dell’Osso, et al., 2008; Kyrios et al., 2004).

Otra variable relevante en el desarrollo del consumo en la adolescencia es el nivel de materialismo, dado que diversos estudios señalan la presencia de una relación directa entre la presencia de valores materiales y la compra impulsiva (Denegri, Sepúlveda et al., 2014; Poeschl, 2015; Yeniaras, 2016) y entre compra compulsiva y nivel de materialismo o valores materiales (Bushra y Bilal ,2014; Dittmar et al., 2007; Zampieri et al., 2011). Al respecto, Richins y Dawson (1992) definen al materialismo o presencia de valores materiales como el conjunto de creencias centrales que un individuo tiene sobre la importancia de las posesiones materiales en la vida. Así, las personas con altos valores materiales no sólo se enfocan en la posesión y mantenimiento de bienes, sino que también construyen creencias sobre los beneficios psicológicos que estos les pueden proporcionar (Dittmar et al., 2007; Kasser et al., 2007).

Algunos estudios muestran que un alto nivel de materialismo impactaría negativamente en la satisfacción con la vida definida ésta como la evaluación global que realizan las personas al comparar sus circunstancias actuales con un patrón cultural que consideran se ajusta a su condición. Los estudios indican que la satisfacción con la vida tiene una relación directa con el estado de salud, la familia, las relaciones interpersonales y la situación socioeconómica, entre otras (Omar et al., 2009; Schnettler et al. 2014; Vásquez et al., 2012).

En términos etarios, si bien algunos estudios indican una reducción gradual en la percepción de satisfacción con la vida a medida que se avanza en la adolescencia y aumenta la capacidad crítica y autocrítica de los jóvenes (Castellá et al., 2012), otros estudios muestran que tanto los niños como los 
adolescentes refieren altos niveles de satisfacción vital (Proctor et al., 2009). En cuanto a la influencia del materialismo en la satisfacción con la vida, éste influiría generando mayor discrepancia entre el "yo real" y el "yo ideal" en relación con la presencia de las posesiones como fuente de satisfacción. Esta discrepancia sería más fuerte cuando las aspiraciones de poder adquisitivo y de estatus social se alejan considerablemente del poder adquisitivo real y de las condiciones objetivas de vida, situación muy frecuente en los segmentos de menores ingresos (Chan y Prendergast, 2007; Howell y Hill, 2009; Howell, et al., 2012; Kasser et al., 2014).

Con base en la revisión del estado del arte y los antecedentes empíricos expuestos, se desprenden los objetivos e hipótesis que guían a este estudio:

Objetivo General. Examinar la relación entre estilos de compra, valores materiales y satisfacción con la vida en adolescentes que cursan educación secundaria y que pertenecen a distintos niveles socioeconómicos del sur de Chile.

Objetivos Específicos: 1) Describir los estilos de compra, valores materiales y satisfacción con la vida en adolescentes que cursan educación secundaria y que pertenecen a distintos niveles socioeconómicos del sur de Chile; 2) Identificar las relaciones existentes entre estilos de compra, valores materiales y satisfacción con la vida en los participantes del estudio, y 3) Evaluar si existen diferencias en los puntajes de los estilos de compra, valores materiales y en las dimensiones de satisfacción vital de acuerdo al nivel socioeconómico de los participantes.

De acuerdo con la revisión y los resultados de la literatura previa, se espera que:

H1. La presencia de valores materiales (materialismo) estará relacionada positiva y directamente con la compra impulsiva.

H2. La presencia de valores materiales (materialismo) estará relacionada positiva y directamente con la compra compulsiva.

H3. Se observará una relación inversa entre satisfacción vital y la presencia de valores materiales (materialismo).

H4. Se observara una relación directa y positiva entre compra impulsiva y compulsiva.

H5. Existirán diferencias significativas en las dimensiones de la satisfacción con la vida de acuerdo al nivel socioeconómico de los participantes.

H6. Se observarán diferencias significativas en el puntaje de compra impulsiva de acuerdo al nivel socioeconómico de los participantes.

H7. Se observarán diferencias significativas en los puntajes de valores materiales (materialismo) de acuerdo al nivel socioeconómico de los participantes.

\section{Método}

El muestreo fue bietápico estratificado por conglomerados, (Barreiro y Albandoz, 2001). La unidad muestral en la primera etapa la constituyeron los establecimientos educativos, y en la segunda los grupos-curso. Para ello, en la primera etapa se seleccionaron establecimientos educativos que cumplieran los siguientes criterios de inclusión: 1) matrícula mixta, 2) localización urbana, 3) que tuvieran más de un curso de 10. y 20. año de educación Secundaria, 4) que la asignación de los estudiantes a los cursos fuera heterogénea y, finalmente 5) que registraran un índice de vulnerabilidad educacional (IVE) entre 2 desviaciones típicas hacia arriba o hacia abajo del promedio nacional. Este último criterio permitió la determinación de nivel socioeconómico utilizando como referente la medición anual de los establecimientos educativos realizada por el Ministerio de Educación de Chile, donde a mayor índice de 
vulnerabilidad, nivel socioeconómico más bajo (Junta Nacional de Auxilio Escolar y Becas [JunAEB], 2005).

En la segunda etapa se consideraron como conglomerados a los cursos completos a los que pertenecían los estudiantes, los que fueron seleccionados al azar entre los cursos de los niveles de 1 o. y 20 . de cada establecimiento, quedando en cada caso un curso de cada nivel. Así, la muestra quedó conformada por 11 cursos de 10. y 20. año de educación Secundaria constituidos por 423 adolescentes de diferentes establecimientos de una comuna urbana del sur de Chile. El promedio de edad fue de 15.62 años (DT = 2.14); $54.4 \%$ de la muestra fueron mujeres y $45.6 \%$ hombres, y la distribución por nivel socioeconómico según IVE fue de Nivel Medio Bajo (54.9\%), Medio (34.2\%) y Medio Alto (7.9\%). Considerando un tamaño poblacional de 54,573 estudiantes (Ministerio de Educación de Chile, 2014), la muestra es representativa con un nivel de confianza de $95 \%$ y un porcentaje de error del $5 \%$. Se utilizó un diseño no experimental, correlacional-transversal (Sampieri et al., 2014).

Los instrumentos empleados fueron:

1) Escala de actitudes hacia la compra (Luna-Arocas y Ferres, 1998, adaptada para adolescentes por Denegri et al., 2014). Consta de 18 ítems en formato tipo Likert (de 1 a 6) y posee tres dimensiones: compra planificada o cognitiva, compra impulsiva y compra compulsiva. Para el presente estudio, la confiabilidad del instrumento mediante a de Cronbach indicó 0.83 para la dimensión compra planificada, 0.86, para compra impulsiva y 0.90 para compra compulsiva.

2) Escala de materialismo para adolescentes (Goldberg et al., 2003), compuesta por 10 ítems en formato tipo Likert (del 1 al 6) que reflejan la importancia de los bienes materiales para los adolescentes. Su confiabilidad para este estudio fue a 0.79 .

3) Escala breve de satisfacción con la vida para estudiantes (BMSLSS) (Selingson et al., 2003). Contiene 5 ítems en formato Likert (del 1 al 7) que evalúan 5 dominios específicos de satisfacción con la vida: familia, amistades, escuela, satisfacción con el "yo" y lugar de residencia. En el estudio el instrumento mostró un a de 0.77.

Se elaboró una lista de colegios que cumplieran los criterios de inclusión ya descritos y se seleccionó al azar un tercio de ellos, a los cuales se envió una carta de invitación a su director describiendo el objetivo del estudio y solicitando autorización para ejecutarlo. Una vez recibidas las aceptaciones se sorteó en cada establecimiento un curso para cada nivel de 10. y 20. medio, considerando a los cursos completos como conglomerados. Una vez realizado el sorteo se solicitó al establecimiento la lista de los estudiantes por cada curso seleccionado y se envió un formato de consentimiento informado a los padres de los participantes para que autorizaran la participación de sus hijos en el estudio. Una vez recibido el consentimiento se contactó a los estudiantes para solicitar participación. Los instrumentos fueron aplicados por encuestadores entrenados (estudiantes de Psicologia del último curso de la carrera) al curso completo en una sola sesión que tomó entre 30 y 45 minutos.

Para el análisis de datos se utilizó el paquete estadístico SPSS v.20. Se utilizaron estadísticos descriptivos рага conocer las medias de las variables considerando como referencia para su interpretación la media teórica de cada escala. Para medir la confiabilidad de las escalas se utilizó la prueba Alfa de Cronbach mientras que para examinar las relaciones entre las variables se utilizaron correlaciones $r$ de Pearson. Esta medida fue utilizada específicamente para examinar la relación entre materialismo y compra impulsiva (H1), materialismo y compra compulsiva $(\mathrm{H} 2)$, entre compra impulsiva y compulsiva $(\mathrm{H} 3)$, y la relación inversa entre satisfacción con la vida y materialismo $(\mathrm{H} 4)$.

Para examinar la relación entre nivel socioeconómico y satisfacción con la vida, compra planificada, compra impulsiva y satisfacción vital $(\mathrm{H} 5, \mathrm{H} 6, \mathrm{H} 7$ y H8) se hizo un análisis de tendencia lineal a partir de la prueba ANOVA unifactorial intersujetos. 


\section{Resultados}

Рara el análisis descriptivo de medias de cada una de las escalas se establecieron como factores relevantes aquellos con una media superior a la media métrica teórica de la escala. En el caso de las escalas de Materialismo, Compra Planificada, Compra Impulsiva y Compra Compulsiva fue de 3.5. Para la escala de Satisfacción con la vida la media teórica fue de 4.0.

Como puede observarse en la tabla 1, las medias de Compra Planificada y Compra Impulsiva se ubican en la media teórica mientras que en Satisfacción con la vida se observa una media bastante más alta que la media teórica, con un 6.5\% de participantes que se ubican bajo la media teórica. Por su parte, Materialismo se ubica bajo la media teórica con un $25 \%$ de participantes que se ubican sobre ella. Lo mismo sucede con Compra Compulsiva, donde sólo un $8 \%$ de los estudiantes se ubican sobre la media teórica.

Tabla I. Medias obtenidas en las variables medidas

\begin{tabular}{l|c|c|c|c|c}
\hline & N & Mínimo & Máximo & Media & $\begin{array}{c}\text { Desviación } \\
\text { estándar }\end{array}$ \\
\hline Materialismo & 423 & 1.00 & 5.50 & 3.0164 & .81631 \\
Compra planificada & 423 & 1.00 & 6.00 & 3.5269 & 1.36361 \\
Compra impulsiva & 423 & 1.00 & 6.00 & 3.5014 & 1.14992 \\
Compra compulsiva & 422 & 1.00 & 6.00 & 1.8302 & 1.00794 \\
Satisfacción con la vida & 423 & 2.60 & 7.00 & 5.7551 & .89428 \\
N válido (por lista) & 423 & & & & \\
\hline
\end{tabular}

\subsection{Correlación de las variables}

En este apartado se exponen los resultados correspondientes a las hipótesis 1, 2, 3 y 4. Como se observa en la tabla II, en relación a H1 los participantes que presentaron un alto puntaje en valores materiales presentaron también un alto puntaje en compra impulsiva, con una correlación estadísticamente significativa, directa y de moderada intensidad $(r=0.50, p<0.01)$.

Asimismo, tal como se predecía en $\mathrm{H} 2$, se observa una relación directa entre valores materiales y compra compulsiva con moderada intensidad $(r=0.44, p<0.01)$.

En cuanto a la $\mathrm{H} 3$, los resultados muestran una relación inversa y de baja intensidad $(r=-0.23, p<0.01)$ entre satisfacción con la vida y compra compulsiva. En esta misma dirección y de acuerdo a lo predicho en la H4, se observa una relación inversa y de baja intensidad entre satisfacción con la vida y valores materiales $(r=-0.11, p=0.012)$.

Por otra parte, en relación a H5, los participantes que mostraron altos puntajes en compra impulsiva, también mostraron altos puntajes en compra compulsiva, indicando una relación directa y de moderada intensidad $(r=0.49, p<0.01)$ entre ambas. 
Tabla II. Correlación entre las variables en estudio

\begin{tabular}{cccccc}
\hline & $\begin{array}{c}\text { Valores } \\
\text { materiales } \\
(1)\end{array}$ & $\begin{array}{c}\text { Compra } \\
\text { planificada } \\
(2)\end{array}$ & $\begin{array}{c}\text { Compra } \\
\text { impulsiva } \\
(3)\end{array}$ & $\begin{array}{c}\text { Compra } \\
\text { compulsiva } \\
(4)\end{array}$ & $\begin{array}{c}\text { Satisfacción } \\
\text { vital } \\
(5)\end{array}$ \\
\hline $\begin{array}{c}\text { Valores materiales } \\
(1)\end{array}$ & 1 & $-.110 *$ & $.500 * *$ & $.440 * *$ & $-.110^{* *}$ \\
$\begin{array}{c}\text { Compra planificada } \\
(2)\end{array}$ & $-.109 *$ & 1 & .045 & .081 & -.006 \\
$\begin{array}{c}\text { Compra impulsiva } \\
(3)\end{array}$ & $.499 * *$ & .045 & 1 & $.490 * *$ & .031 \\
$\begin{array}{c}\text { Compra compulsiva } \\
(4)\end{array}$ & $.460 * *$ & .081 & $.525 * *$ & 1 & $-.230 * *$ \\
$\begin{array}{c}\text { Satisfacción vital } \\
(5)\end{array}$ & $-.124 * *$ & -.006 & .031 & $-.180 * *$ & \\
\hline$* 0<0.05$ & & & & & \\
$*{ }^{* *} p<0.01$
\end{tabular}

\subsection{Diferencias por nivel socioeconómico}

En este apartado se exponen los resultados correspondientes a las hipótesis 5,6, y 7. Los resultados del análisis de tendencia lineal, a partir de la prueba ANOVA unifactorial intersujetos muestran que no existe una relación estadísticamente significativa entre ninguna de las dimensiones de la satisfacción con la vida y nivel socioeconómico rechazándose H5.

En cuanto a H6, como se observa en la figura 1, existe una relación lineal entre compra impulsiva y nivel socioeconómico Flineal $=6.990 p=0.009$, Eta2 $=0.016$.

Figura 1. Relación lineal entre compra impulsiva y nivel socioeconómico

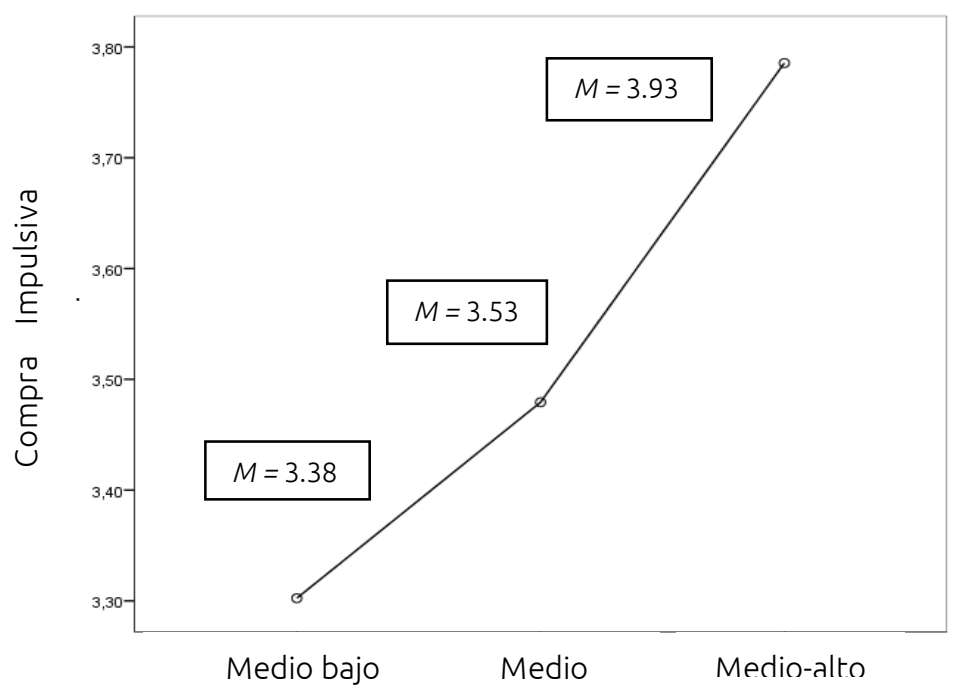

Por último, con relación a $\mathrm{H} 7$ no se observan diferencias estadísticamente significativas en los valores materiales (materialismo) de acuerdo al nivel socioeconómico, por lo que se rechaza H8. 


\section{Discusión}

Este estudio pretendía examinar la relación entre estilos de compra, valores materiales y satisfacción con la vida en adolescentes de educación secundaria de diversos niveles socioeconómicos del sur de Chile.

Con relación a la presencia de valores materiales los resultados indican niveles moderados de materialismo en la muestra con un $25 \%$ de participantes que se ubicarían sobre la media teórica de la escala y, por lo tanto, exhibirían un alto nivel de materialismo. La presencia general de bajos niveles de valores materiales podría vincularse a las características del desarrollo de la adolescencia y su proceso aún en desarrollo de construcción de identidad, en el cual los valores juegan un papel relevante, pero aún no se encuentran consolidados. Este resultado concuerda con lo encontrado por Pizzutti y Heyde (2011), quienes señalan la coexistencia de actitudes favorables y desfavorables frente al materialismo en los adolescentes, con un rechazo al autorreconocimiento como "materialistas" a pesar de mostrar comportamientos de alta valoración de los objetos materiales como forma de diferenciación y, a la vez, reconocimiento entre sus pares. Este es un tema que deberá ser profundizado en investigaciones posteriores.

En cuanto a compra planificada y compra impulsiva, los adolescentes muestran niveles medios y altos en ambas variables, un $50 \%$ de los encuestados se ubican sobre la media teórica. Llama la atención la coexistencia de ambos estilos de consumo, lo cual podría orientar a una fragilidad y ambivalencia en la forma en que estos jóvenes se enfrentan a sus decisiones de compra y, por ello, al riesgo de mal manejo financiero (Denegri et al., 2012).

En satisfacción con la vida se observan medias muy altas en toda la muestra estudiada y para todas las dimensiones de la escala, lo cual es consistente con lo encontrado por Proctor et al. (2009) en su revisión de estudios sobre satisfacción vital en jóvenes; según estos autores -y coincidente con nuestros resultados- la mayoría de los estudios muestran que tanto los niños como los adolescentes refieren altos niveles de satisfacción vital.

En cuanto a las relaciones hipotetizadas entre las variables Valores materiales, Estilos de compra y Satisfacción con la vida, los resultados permiten aceptar las cinco variables formuladas, ya que muestran que existe una relación directa entre valores materiales, compra impulsiva e compulsiva, confirmando lo encontrado en estudios previos en poblaciones juveniles (Poeschl, 2015; Yeniaras, 2016). Estos resultados reflejan el impacto de la idealización de los bienes materiales propia de las características de las actuales sociedades de consumo y de la transición en la sociedad chilena, desde la austeridad hacia una sociedad altamente hedonista que idealiza el acceso a las posesiones de manera inmediata como una autoafirmación del yo (Denegri, 2010). En el caso del segmento de adolescentes que presenta alto materialismo se podría hipotetizar que ello también podría aumentar la probabilidad de mayor impulsividad en las compras, desarrollando un estilo que, de mantenerse en su vida adulta, pondría en riesgo su estabilidad financiera (Dell'Osso et al., 2008). Este es un tema que debería explorarse en futuras investigaciones que se orienten al levantamiento de perfiles diferenciales en cuanto a la estructura de relaciones de estas variables en los adolescentes.

Por otra parte, la Satisfacción vital guarda una relación inversa con los Valores materiales, hallazgo que resulta coherente con lo postulado por Xiao et al. (2009). Esto debe examinarse a la luz de la brecha que se genera a nivel psicológico entre el "yo real" y el "yo ideal" propio de la adolescencia, donde existiría una contradicción entre las aspiraciones y el poder adquisitivo real y esto afectaría el nivel de satisfacción con la vida (Howell et al., 2012; Kasser et al., 2014). Asimismo, los resultados indican que la satisfacción con la vida se relaciona inversamente con la compra compulsiva, lo que es consecuente con la literatura previa. (Dell'Osso et al., 2008; Kyrios et al., 2004).

En torno a las hipótesis que planteaban relaciones entre satisfacción con la vida, y nivel socioeconómico, los resultados indican que no existen relaciones estadísticamente significativas entre ninguna de las dimensiones de la Satisfacción con la vida y el nivel socioeconómico de los participantes. Si bien esto contradice algunos resultados encontrados en adultos y adultos mayores que indican que el nivel 
socioeconómico marca diferencias importantes, en el nivel socioeconómico alto se observan mayores niveles de Satisfacción con la vida en contraposición con el nivel socioeconómico bajo (Castillo et al., 2012; Martínez, 2004), esto concuerda con estudios nacionales donde más del 85\% de los jóvenes se declaran satisfechos con diferentes aspectos de sus vidas y del futuro personal (Instituto Nacional de la Juventud, 2017).

En cuanto a la relación entre Compra impulsiva y nivel socioeconómico, se aprecia que a medida que aumenta el nivel socioeconómico aumenta también la compra impulsiva. Un elemento que podría estar explicando estas diferencias es el mayor acceso al dinero que tienen los adolescentes de los niveles medio y medio alto en comparación con sus pares de nivel medio bajo y la libertad que tienen para utilizarlo (Orcasita y Uribe, 2010; Lai, 2010).

Finalmente, no se evidencian diferencias en valores materiales por nivel socioeconómico, lo que señala la transversalidad de la penetración de la sociedad de consumo y sus aspiraciones en todos los segmentos de la población (Twenge y Kasser, 2013).

Como proyección de los resultados obtenidos, en futuras investigaciones habría que dilucidar qué variables moderan y median las relaciones encontradas en este estudio. Asimismo, sería relevante investigar la incidencia de los agentes de socialización, como la familia y grupos de pares, en la forma en que las pautas de consumo son transmitidas, interiorizadas y reproducidas por este segmento poblacional.

\section{Referencias}

Barreiro, L. P. y Albandoz, J. P. (2001). Población y muestra. Técnicas de muestreos. Management Mathematics for European Schools. https://www.yumpu.com/es/document/read/14117689/ poblacion-ymuestra-tecnicas-de-muestreos

Bushra, A. y Bilal, A. (2014). The relationship of compulsive buying with consumer culture and postpurchase regret. Pakistan Journal of Commerce y Social Sciences, 8(3), 590-611.

Castellá, J., Saforcada, E., Tonón, G., Rodríguez, L., Mozobancyk, S. y Bedin, L. (2012). Bienestar subjetivo de los adolescentes: un estudio comparativo entre Argentina y Brasil. Intervención Psicosocial, 21(3), 273 280.

Castellanos, L. M., Sepúlveda, J. y Denegri, M. (2016). Análisis teórico de la relación entre estilos de compra, valores materiales y satisfacción con la vida en la adolescencia. Revista de Psicología y Ciencias Del Comportamiento, 7(1), 1-22.

Castillo, A., Albala, C., Dangour, A. y Uauy, R. (2012). Factores asociados a satisfacción vital en una cohorte de adultos mayores de Santiago, Chile. Gaceta Sanitaria (26)5, 414-420.

Chan, K. y Prendergast, G. (2007). Materialism and social comparison among adolescents. Social Behavior and Personality, 35(2), 213-228. http://doi.org/10.2224/sbp.2007.35.2.213

Dell'Osso, B., Allen, A., Altamuro, C., Buoli, M. y Hollander, E. (2008). Impulsive-compulsive buying disorder: clinical overview. The Royal Australian and New Zealand College of Psychiatrists, 42, 259-266.

Denegri, M. (2010). Introducción a la psicología económica. Psicom.

Denegri, M. y Martínez, G. (2004). ¿Ciudadanos o consumidores? Aportes constructivista a la educación para el consumo. PAIDEA Revista de Educación 37, 101-116. 
Denegri, M., Alí I., Novoa, M., Rodríguez, C., Del Valle, C., González, Y., Etchebarne, M., Miranda, H. y Sepúlveda, J. (2012). Relaciones entre las escalas actitudes hacia el dinero y la compra: un estudio en estudiantes de pedagogía de Chile. Revista Interamericana de Psicología, 46(2), 229-238.

Denegri, M., García, C., González, N. y Sepúlveda, J. (2014). ¿Educadores o consumidores? Discrepancia del yo, consumo y valores materiales en estudiantes chilenos de pedagogía. Actualidades Investigativas en Educación, 14(2), 342-372.

Denegri, M., Sepúlveda, J., González, T., Romero, G., Ulloa, J. y Vásquez, D. (2014). Actitudes hacia el consumo y materialismo en estudiantes universitarios de pedagogía en Chile. Fronteras, 1(2), 45-62.

Dittmar, H., Long, K. y Bond, R. (2007). When a better self is only a button click away: associations between materialistic values, emotional and identity-related buying motives, and compulsive buying tendency online. Journal of Social y Clinical Psychology, 26(3), 334-361.

https://doi.org/10.1521/iscp.2007.26.3.334

Gempp, R., Denegri, M., Caripán, N., Catalán, V., Hermosilla, S. y Caprile, C. (2007). Desarrollo del Test de Alfabetización Económica para Adultos TAE-A-25. Interamerican Journal of Pshychology, 41(3), 275-284.

Goldberg, M. E., Gorn, G. J., Peracchio, L. A. y Bamossy, G. (2003). Understanding materialism among youth. Journal of Consumer Psychology, 13, 278-288. https://doi.org/10.1207/S15327663JCP1303 09

Hernández-Sampieri, R., Fernandez-Collado, C. y Baptista-Lucio, P. (2014). Metodología de la Investigación. McGraw-Hill.

Howell, R. T. y Hill, G. (2009). The mediators of experiential purchases: determining the impact of psychological needs satisfaction and social comparison. The Journal of Positive Psychology, 4, 511-522.

Howell, R. T., Pchelin, P. e Iyer, R. (2012). The preference for experiences over possessions: measurement and construct validation of the experiential buying tendency scale. The Journal of Positive Psychology, 7(1), 57-71. https://doi.org/10.1080/17439760.2011.626791

Instituto Nacional de la Juventud. (2017). Octava Encuesta Nacional de Juventud.

http://www.injuv.gob.cl/storage/docs/INFORME ARAUCANIA web.pdf

Junta Nacional de Auxilio Escolar y Becas. (2005). Sistema Nacional de Asignación con Equidad para Becas JUNAEB. https://www.junaeb.cl/wp-content/uploads/2013/02/libro junaeb.pdf

Jurado, D., Sejnaui, P. y Uribe-Rodríguez, A. (2011). Impulsividad en la compra en estudiantes universitarios. Revista de Psicología Universidad de Antioquia, 3(2), 25-38.

https://aprendeenlinea.udea.edu.co/revistas/index.php/psicologia/article/view/11366

Kasser, T., Rosenblum, K., Sameroff, A., Deci, E., Niemiec, C., Ryan, R. y Hawks, S. (2014). Changes in materialism, changes in psychological well-being: evidence from three longitudinal studies and an intervention experiment. Motivation \& Emotion, 38(1), 1-22. http://doi.org/10.1007/s11031-013-9371-4

Kasser, T., Cohn, S., Kanner, A. y Ryan, R. (2007). Some costs of American corporate capitalism: a psychological exploration of value and goal conflicts. Psychological Inquiry, 18(1), 1-22.

https://doi.org/10.1080/10478400701386579

Kyrios, M., Frost, R. O. y Steketee, G. (2004). Cognitions in compulsive buying and acquisition. Cognitive Therapy and Research, 28(2), 241-258. https://doi.org/10.1023/B:COTR.0000021543.62799.32 
Lai, C. (2010). How financial attitudes and practices influence the impulsive buying behavior of college and university students. Social Behavior and Personality, 38(3), 373-380.

https://doi.org/10.2224/sbp.2010.38.3.373

Luna-Arocas, R. y Fierres, R. (1998). Incidencia en la compra por impulso en la ciudad de Valencia. Revista investigación y marketing, 60, 36-42.

Luna, R. y Quintanilla, I. (2000). Modelo de compra ACB, una nueva conceptualización de la compra por impulso, Esic Market, 101, 151-163.

https://www.esic.edu/documentos/revistas/esicmk/060130975593 E.pdf

Luna, R. Puello, S. y Botero, M. (2004). La compra impulsiva y el materialismo en los jóvenes: estudio exploratorio en estudiantes universitarios de Barranquilla (Colombia). Psicología desde el Caribe, 14, 1-26. http://rcientificas.uninorte.edu.co/index.php/psicologia/article/viewFile/1777/1157

Martínez, P. (2004). Perspectiva temporal futura y satisfacción con la vida a lo largo del ciclo vital. Tesis doctoral, Universitat Autònoma de Barcelona. https://ddd.uab.cat/record/37008

Ministerio de Educación (2014). Estadísticas de la Educación 2014. https://centroestudios.mineduc.cl/wpcontent/uploads/sites/100/2017/06/Estad\%C3\%ADsticas-de-la-Educaci\%C3\%B3n-2014.pdf

Omar, A., Paris, L., Aguilar, M., Almeida, S. y Del Pino, M. (2009). Validación del inventario de bienestar subjetivo con muestras de jóvenes y adolescentes argentinos, brasileros y mexicanos. Suma Psicológica, 16(2), 69-84.

Orcasita, L. T. y Uribe, F. (2010). La importancia del apoyo social en el bienestar de los adolescentes. Psychologia Avances de la Disciplina, 4(2), 69-82.

Pizzutti, C. y Heyde, D. (2011). A socialização de consumo ea formação do materialismo entre os adolescentes [La socialización del consumidor y la formación del materialismo entre los adolescentes]. Revista de Administração Mackenzie, 12(1), 169-203.

Poeschl, G. (2015). O papel do materialismo como mediador entre o prazernas compras e a compra por impulso. [El papel del materialismo como mediador entre Prazernas Compras y la compra por impulso]. Psico, 46(1), 57-67. http://doi.org/10.15448/1980-8623.2015.1.16864

Proctor, C. L., Linley, P. A. y Maltby, J. (2009). Youth life satisfaction: a review of the literature. Journal of Happiness Studies, 10, 583-630. https://doi.org/10.1007/s10902-008-9110-9

Richins, M. y Dawson, S. (1992). Materialism as a consumer value: measure development and validation. Journal of Consumer Research, 19, 303-316.

Rook, D. W. y Fisher, R. J. (1995). Normative influences on impulsive buying behavior. Journal of Consumer Research, 22(3), 305-313. https://doi.org/10.1086/209452

Schnettler, B., Miranda, H., Sepúlveda, J., Orellana, L., Denegri, M., Mora, M. y Lobos, G. (2014). Variables que influyen en la satisfacción con la vida de personas de distinto nivel socioeconómico en el sur de Chile. Suma Psicológica, 21(1), 54-62.

Seligson, J. L., Huebner, E. S. y Valois, R. F. (2003). Preliminary validation of the Brief Multidimensional Students' Life Satisfaction Scale (BMSLSS). Social Indicators Research, 61(2), 121-145.

Shim, S., Serido, J. y Barber, B. (2011). A consumer way of thinking linking consumer socialization and consumption motivation perspectives to adolescent development. Journal of Research on Adolescence, 21(1), 290-299. https://doi.org/10.1111/j.1532-7795.2010.00730.x 
Twenge J. M. y Kasser T.(2013) Generational changes in materialism and work centrality, 1976-2007: associations with temporal changes in societal insecurity and materialistic role modeling. Personality and Social Psychology Bulletin, 39(7), 883-97. http://doi.org/10.1177/0146167213484586

Vásquez, C., Duque, A. y Hervas, G. (2012). Escala de Satisfacción con la Vida (swLS) en una muestra representativa de españoles adultos: validación y datos normativos. The Spanish Journal of Psychology, 15(1), 1-37. https://doi.org/10.1017/sjp.2013.82

Vogt, S., Hunger, A., Pietrowsky, R. y Gerlach, A. L. (2015). Impulsivity in consumers with high compulsive buying propensity. Journal of Obsessive-Compulsive and Related Disorders, 7, 54-64. http://doi.org/10.1016/j.jocrd.2015.10.002

Xiao, J. J., Tang, C. y Shim, S. (2009). Acting for happiness: financial behavior and life satisfaction of college students. Social Indicators Research, 92, 53-68. http://doi.org/10.1007/s11205-008-9288-6

Yeniaras, V. (2016). Uncovering the relationship between materialism, status consumption and impulsive buying: newfound status of Islamists in turke. Journal for the Study of Religions and Ideologies, 15(44), 153.

Zampieri, M., Flores, L., Beuron, T. A., Riss, L. A., Carpes, A. y Lutz, C. (2011). Relação entre materialismo e estilo de consumo: homens e mulheres com comportamento díspare? [Relación entre materialismo y estilo de consumo: ¿hombres y mujeres con comportamientos diferentes?]. Contaduría y Administración, 57(1), 185-214. 